\title{
Ab Initio Calculation of UV-Vis Absorption Spectra of a Single Chlorophyll $a$ Molecule: Comparison Study between RHF/CIS, TDDFT, and Semi-Empirical Methods
}

\author{
Veinardi Suendo ${ }^{1,3} \&$ Sparisoma Viridi $^{2,3}$ \\ ${ }^{1}$ Inorganic and Physical Chemistry Research Division, Department of Chemistry, \\ Faculty of Mathematics and Natural Sciences, Institut Teknologi Bandung, \\ Jalan Ganesha 10, Bandung, Jawa Barat 40132, Indonesia \\ ${ }^{2}$ Nuclear Physics and Biophysics Research Division, Department of Physics, \\ Faculty of Mathematics and Natural Sciences, Institut Teknologi Bandung, \\ Jalan Ganesha 10, Bandung, Jawa Barat 40132, Indonesia \\ ${ }^{3}$ National Center for Nanotechnology, Jalan Ganesha 10, Bandung, \\ Jawa Barat 40132, Indonesia \\ Email: vsuendo@chem.itb.ac.id
}

\begin{abstract}
Chlorophyll $a$ is the most abundant pigment on Earth responsible for trapping light energy to perform photosynthesis in green plants. This molecule has been studied for many years from different points of interest with both experimental and theoretical methods. In this study, the Restricted Hartree-Fock /Configuration Interaction Single (RHF/CIS), Time-Dependent Density Functional Theory (TDDFT), and several semi-empirical methods (CNDO/S and ZINDO) calculations were carried out to reconstruct the UV-Vis absorption spectra of chlorophyll $a$. To some extent, the calculation results based on the single-molecule approach succeeded to reconstruct the absorption spectra, but they required to be rescaled to fit the experimental results. In general, the semiempirical methods provide a better energy scaling factor. However, they lack vertical transition fine features with respect to the spectrum obtained experimentally. In this case, the ab initio calculations provided more complete features, especially the TDDFT with high-level basis sets, which also has a good accuracy with regards to the transition energies. The contribution of the ground state and excited state orbitals in the main vertical transitions is discussed based on the delocalized nature of the wave functions and the presence of solvents using the polarizable continuum model (PCM).
\end{abstract}

Keywords: Chlorophyll a; CIS; CNDO/S; Electronic transition; TDDFT; UV-Vis absorption spectra; ZINDO.

\section{$1 \quad$ Introduction}

Chlorophyll $a$ is the most abundant pigment on Earth responsible for trapping light energy to perform photosynthesis. This molecule is a metal-complex that consists of a porphyrin ring that acts as a ligand with magnesium as the central ion (Figure 1). Chlorophyll $a$ has been studied for many years from different

Received December $22^{\text {nd }}, 2010$, Revised June $7^{\text {th }}, 2011$ Accepted for publication January $26^{\text {th }}, 2012$.

Copyright (C) 2012 Published by LPPM ITB, ISSN: 1978-3043, DOI: 10.5614/itbj.sci.2012.44.2.1 
points of interest with both experimental and theoretical methods. Its intrinsic spectroscopic, magnetic and electrochemical properties have induced many researchers to carry out experiments and studies on the applications of this natural pigment. On the other hand, the molecular model of this compound, a porphyrin metal complex or metal porphyrin, has a structure with a high $D_{4 h}$ molecular symmetry, which is very interesting from a theoretical point of view. To some extent, theoretical studies of the molecular model can explain the general features of metal porphyrins. However, the influence of the attached peripheral groups, especially those that break the symmetry, as in chlorophyll $a$, play an important role in their function. These influences can be seen clearly in how the absorption/emission spectra of chlorophyll $a$ differ from chlorophyll $b$, while both of them have the same main features.

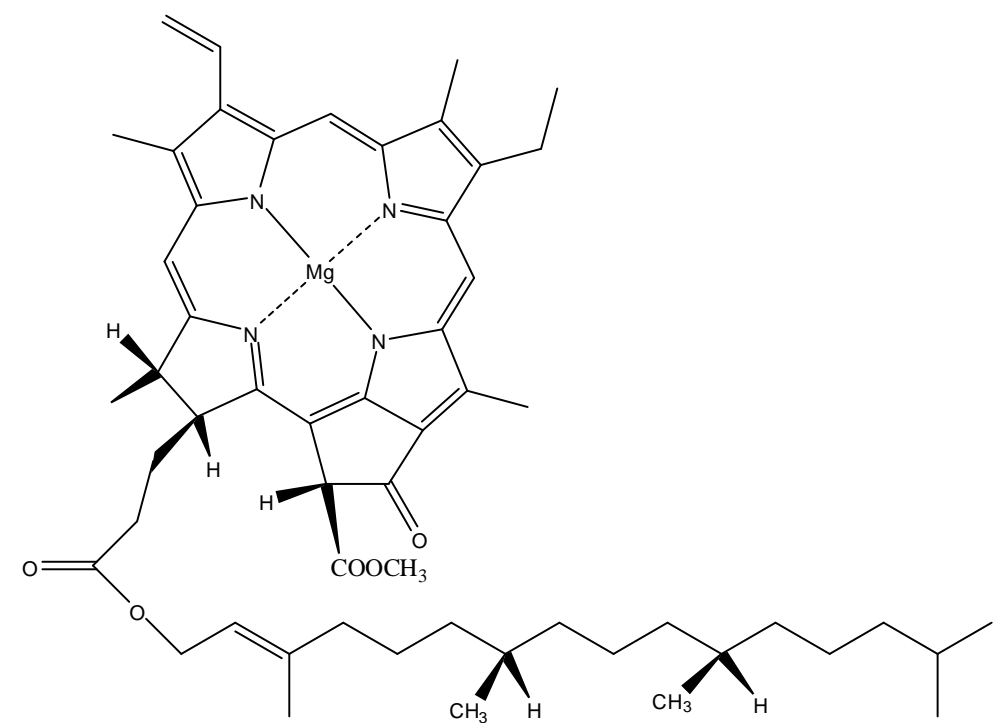

Figure 1 Structural formula of the chlorophyll $a$ molecule.

The ability of this molecule to convert light into electricity and to induce redox reactions is very interesting with respect to its application in artificial systems. Thus, this molecule is a prospective candidate for a base material in many new environmentally friendly devices, such solar cells [1] or light emitting diodes [2]. The main issues concerning the implementation of this molecule in real life are the efficiency and stability of the molecule itself when functioning in a device. These properties depend to a large degree on the nature of both the ground state and the excited state orbitals. Thus, knowing the electronic states of this molecule will reveal a better understanding about their functions related to the electronic excitation-deexcitation processes involved in the ground state and excited state orbitals in various environments, e.g. in solution, in photosynthetic light-harvesting protein complexes and in well-functioning 
electronic devices. For example, it is not well understood why the $Q_{x}$ and the Soret bands are much more sensitive to solvent effects than the $Q_{y}$ band [3]. This is a real orbital interaction problem, which has to be solved on a quantum mechanical level, and is rather difficult to study directly with experiments. Thus, in this case an ab initio calculation is one of the best ways to study the excited state and the possible transitions.

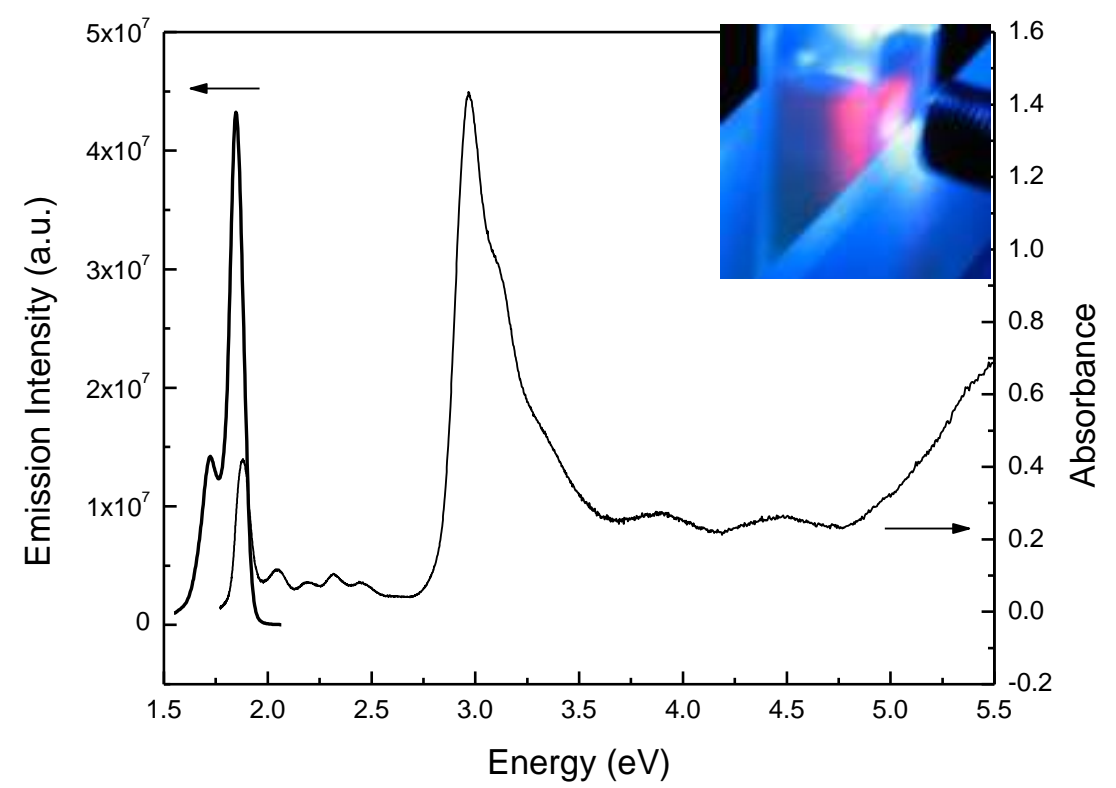

Figure 2 Fluorescence and absorption spectra of chlorophyll $a$ in methanol [4]. The image in the inset shows a strong red emission from the chlorophyll $a$ solution excited near the Soret band at $405 \mathrm{~nm}$.

The experimental spectra of the chlorophyll $a$ molecule consist of two main absorption bands (Figure 2). The more complex one is located in the blue region, and is called the Soret band, which consists of many electronic transitions, with the strongest oscillator strength at $2.88 \mathrm{eV}$, called $B$ transition [3,5]. The other band is found in the red region and is called the $Q$ band, which consists of $Q_{\mathrm{y}}$ and $Q_{x}$ transitions at 1.87 and $2.14 \mathrm{eV}$, respectively [3,5]. The calculated electronic spectra of chlorophyll $a$ have been studied intensively with high accuracy [6-8] and compared with its pheophytin form [8,9]. Here, the calculation results will hopefully help in constructing a better model to optimize the design of new materials and devices. We focus on two calculation methods: the Configuration Interaction Singles (CIS) method, which is well-known as a low-cost calculation method; and the Time-Dependent Density Functional Theory (TDDFT), which is a more reliable method. Here, the basis set that was 
used to construct the molecular wave function is varied at three polarization levels: 6-31G, 6-31G(d) and 6-31G(d,p). For comparison two semi-empirical methods (CNDO/S and ZINDO) were also carried out to calculate the UV-Vis absorption spectra in order to gain a clear perspective of the accuracy of the semi-empirical methods under study.

\section{Methods}

\subsection{Configuration Interaction Singles (CIS) [10,11]}

The CIS calculation can be formulated by starting from the Hartree-Fock (HF) ground state, $\Phi_{0}(\mathbf{r})$, which corresponds to the best single Slater determinant describing the electronic ground state of the system, which can be written as

$$
\Phi_{0}(\mathbf{r})=\left|\phi_{1}(\mathbf{r}) \phi_{2}(\mathbf{r}) \phi_{3}(\mathbf{r}) \ldots \phi_{n}(\mathbf{r})\right|
$$

A closed-shell ground-state electronic configuration is normally assumed to simplify the problem, where the $\phi_{\mathrm{i}}(\mathbf{r})$ corresponds to doubly occupied spatial orbitals, $n=N / 2$, and $N$ is the number of electrons. Here, $\Phi_{0}(\mathbf{r})$ is obtained by solving the time-independent $\mathrm{HF}$ equation, which is given by

$$
\hat{F}(\mathbf{r}) \Phi_{0}(\mathbf{r})=E_{0} \Phi_{0}(\mathbf{r})
$$

In the configuration interaction, the electronic wave function is then constructed as a linear combination of the ground state Slater determinants and so-called excited determinants, which are obtained by replacing the occupied orbitals of the ground state with the virtual ones. If we replace only one occupied orbital $i$ by one virtual orbital $a$ and then include only the Slater determinants $\Phi_{i}^{a}(\mathbf{r})$ in the CI wave function expansion, then we obtain the CIS wave function, $\Psi_{C I S}$, which can be written as

$$
\Psi_{C I S}=\sum_{i a} c_{i}^{a} \Phi_{i}^{a}(\mathbf{r})
$$

The CIS energy can be obtained readily by introducing the CIS wave function, $\Psi_{C I S}$, into the exact time-independent electronic Schrödinger equation,

$$
\hat{H}(\mathbf{r}) \Psi_{C I S}(\mathbf{r})=E_{C I S} \Psi_{C I S}(\mathbf{r})
$$

Finally, one obtains an expression for the excitation energies,

$$
\omega_{C I S}=E_{C I S}-E_{0}
$$




\subsection{Time-Dependent Density Functional Theory [10,12,13]}

The TDDFT calculation can be formulated by the time-dependent Kohn-Sham equation (TD-KS)

$$
i \frac{\partial}{\partial t} \phi_{i}(\mathbf{r}, t)=\left(-\frac{1}{2} \nabla_{i}^{2}+v(\mathbf{r}, t)+\int d^{3} \mathbf{r} \frac{\rho\left(\mathbf{r}^{\prime}, t\right)}{\left|\mathbf{r}-\mathbf{r}^{\prime}\right|}+\frac{\delta A_{x c}[\rho]}{\delta \rho(\mathbf{r}, t)}\right) \phi_{i}(\mathbf{r}, t)=\hat{F}^{K S} \phi_{i}(\mathbf{r}, t)
$$

with

$$
\rho(\mathbf{r}, t)=\rho_{S}(\mathbf{r}, t)=\sum_{i}^{N}\left|\phi_{i}(\mathbf{r}, t)\right|^{2}
$$

Two different strategies can be used to obtain the excitation energies and oscillator strengths by employing the TD-KS. The first possibility is to propagate the TD-KS wave function in time, which is referred to as real-time TDDFT. The second approach is analysis of the linear response of the TD-KS equation, which is used in this study.

For the linear response of the TD-KS equation, let us suppose there exists a time-dependent perturbing potential $v_{1}(\mathbf{r}, t)$, for an oscillating electric field $v_{1}(\mathbf{r}, t)=E_{z} \cos w t$, which is switched on at time $t=t_{0}$. The external potential can be expressed as

$$
v_{e x t}(\mathbf{r}, t)=v_{0}(\mathbf{r})+v_{1}(\mathbf{r}, t)= \begin{cases}v_{0}(\mathbf{r}) & , t \leq t_{0} \\ v_{0}(\mathbf{r})+E_{z} \cos w t, & t>t_{0}\end{cases}
$$

where $v_{0}$ is the Coulomb potential between electrons and nuclei

$$
v_{0}(\mathbf{r})=-\sum_{K}^{N} \frac{Z_{K}}{\left|\mathbf{R}_{K}-\mathbf{r}\right|}
$$

Thus, the first order deviation of the time-dependent density $\rho(\mathbf{r}, t)$ from the unperturbed ground state density $\rho_{0}(\mathbf{r})$ for interacting particles can be written as

$$
\rho(\mathbf{r}, t)-\rho_{0}(\mathbf{r}) \approx \rho_{1}(\mathbf{r}, t)=\int d t^{\prime} \int d \mathbf{r}^{\prime} \chi\left(\mathbf{r}, t, \mathbf{r}^{\prime}, t^{\prime}\right) v_{1}\left(\mathbf{r}^{\prime}, t^{\prime}\right)
$$

with the interaction response function

$$
\chi\left(\mathbf{r}, t, \mathbf{r}^{\prime}, t^{\prime}\right)=\left.\frac{\delta \rho(\mathbf{r}, t)}{\delta v_{e x t}\left(\mathbf{r}^{\prime}, t^{\prime}\right)}\right|_{v_{0}}
$$

while for non-interacting particles we will have 


$$
\rho_{1}(\mathbf{r}, t)=\int d t^{\prime} \int d \mathbf{r}^{\prime} \chi_{s}\left(\mathbf{r}, t, \mathbf{r}^{\prime}, t^{\prime}\right) v_{s, 1}\left(\mathbf{r}^{\prime}, t^{\prime}\right)
$$

with the Kohn-Sham response function

$$
\chi_{s}\left(\mathbf{r}, t, \mathbf{r}^{\prime}, t^{\prime}\right)=\left.\frac{\delta \rho(\mathbf{r}, t)}{\delta v_{s}\left(\mathbf{r}^{\prime}, t^{\prime}\right)}\right|_{v_{s}\left[\rho_{0}\right]}
$$

Here, the poles of the response function (Eq. 11) of the interacting system represent the electronic transition energies.

\subsection{Computational Detail}

The PM3, RHF, RHF/CIS and TDDFT calculations of chlorophyll $a$ were carried out using the PC GAMESS/Firefly program package developed by Alex A. Granovsky and co-workers at the Department of Chemistry, Moscow State University [14]. Semi-empirical quantum calculations of the UV-Vis absorption spectra were carried out as well, using the software packages Winmostar (CNDO/S, with the following parameters: basis SP, no interaction, repulsion integral $=3$, charge $=0$, nuclear repulsion $=2$, PKappa $=0.585$, DKappa $=0.3$, $\mathrm{CI}=60$, and number of excited states $=1)[15,16]$ and Arguslab (ZINDO, with the following parameters: active occupied $\mathrm{MOs}=20$, active virtual MOs $=20$, number of excitation states $=60$ ) [17-19]. The starting structure of chlorophyll $a$ in the CLA_model.pdb file was obtained from the RSCB Protein Data Bank (http://www.rcsb.org/pdb/home/home.do). Here, all calculations of the chlorophyll $a$ molecule were carried out using the structure in the absence of other ligands, such as $\mathrm{H}_{2} \mathrm{O}, \mathrm{NH}_{3}$ or $\mathrm{Cl}$ - in the axial positions, in order to simplify the calculation. This approach is supported by the experimental evidence of the presence of this structure in nature obtained by ESI-MS (electro-spray ionization mass spectroscopy) measurements. The structure was geometrically optimized in two steps, first using a semi-empirical method at the PM3 level, followed by RHF using the 6-31G(d) basis set until a minimum in energy was reached, prior to any further property calculations. The electronic transitions between occupied and unoccupied states were calculated at the theoretical RHF/CIS and TDDFT/B3LYP level using basis sets 6-31G, 6$31 \mathrm{G}(\mathrm{d})$ and $6-31 \mathrm{G}(\mathrm{d}, \mathrm{p})$, with 60 excitation states that result in the UV-Vis absorption spectra and the main orbitals that contribute to the transitions. Calculation of solvation effects on the vertical electronic transition were also carried out, using the SCRF (Self-Consistent Reaction Field) method based on the Kirkwood-Onsager theory of electrostatic solvation [20] and PCM (Polarizable Continuum Model) [21-23] for semi-empirical and ab initio calculations, respectively. 


\section{$3 \quad$ Results and Discussion}

\subsection{Commonly observed transitions}

Figure 2 shows both absorption and fluorescence spectra of chlorophyll $a$, which were obtained experimentally in methanol [4]. In the absorption spectra, we can observe the presence of $B$ (Soret), $Q_{y}$ and $Q_{x}$ transitions at $2.88 \mathrm{eV}, 1.87$ and $2.14 \mathrm{eV}$, respectively [3,4]. Meanwhile, a strong emission is observed around the $Q$ band or in the red region (see inset Figure 2). These features may indicate that the molecular orbitals involved in the $Q$ transitions are responsible for the emission processes. Furthermore, it is also observed that the excitation energies for fluorescence measurements have to be overlapped with the Soret band. This reveals the presence of energy transfer between the states that are involved in the transitions of the $Q$ and the Soret bands. The SAAP (SpinAdapted Antisymmetrized Product) treatment on both the RHF/CIS and TDDFT calculation reveals that the main molecular orbitals that contribute to the electronic transitions are HOMO-1, HOMO, LUMO and LUMO+1, independent of the level of theory and basis sets.

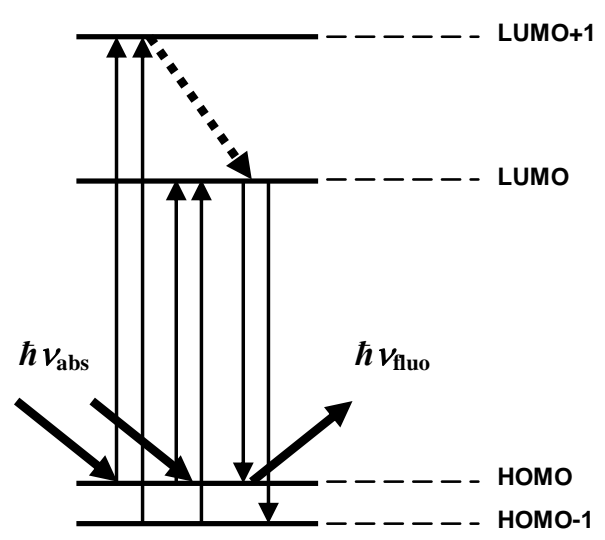

Figure 3 Schematic diagram of excitation and deexcitation processes involving the two highest occupied and the two lowest unoccupied states in the chlorophyll $a$ molecule.

This result is in a good agreement with previously reported results, such as the "four orbitals" scheme [6,24,25] and the shape of the observed UV-Vis absorption spectra presented in Figure 2, that have two main absorption regions representing the $Q$ and the Soret bands, which can be assigned to the transitions from HOMO and/or HOMO-1 to LUMO and LUMO+1, respectively. Thus, the scheme of upward vertical electronic transitions in chlorophyll $a$ can be simplified into four main transitions that are assigned to two absorption bands, $Q$ and Soret, respectively: 


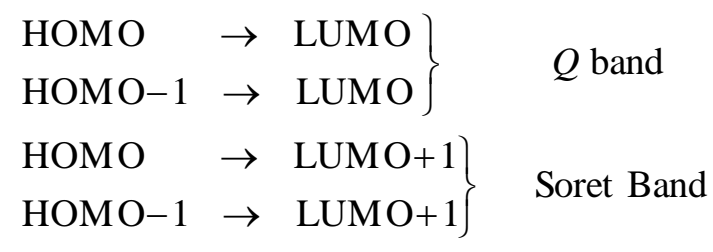

Furthermore, by combining both the experimental data and the calculation results with the Franck-Condon principle, the simplified mechanism for downward vertical transition can be predicted as

$$
\left.\begin{array}{l}
\text { HOMO } \leftarrow \text { LUMO } \\
\text { HOMO-1 } \leftarrow \text { LUMO }
\end{array}\right\} \quad \text { Emission band }
$$

Here, the emission band is located near the $Q$ band, but slightly red shifted due to the relaxation of the excited structure as explained by the Franck-Condon principle. The excited structure has a strong tendency to relax its structure in order to achieve minimum energy before the electrons are deexcited radiatively to the ground state. Figure 3 shows all the simplified vertical electronic transitions of chlorophyll $a$, which cover the $Q$, Soret and emission bands. The downward transition scheme also has a good agreement with the experimental data due to the presence of two main emission features in the observed emission band at 1.72 and $1.85 \mathrm{eV}$ that are assigned to the relaxed form of $Q_{y}$ and $Q_{x}$ transitions, respectively (Figure 2).

Some fine features are also present in the experimental spectra, which appear between the $Q$ and the Soret bands. These features are not so important in some processes, e.g. absorption or emission processes, but they are a very important indicator when selecting a good model for calculating the electronic structure, especially in the study of electronic transitions. They have the opposite behavior compared to the $Q$ or Soret transitions; these fine transitions are very sensitive to the specific wavefunction that is used in the calculation. Their presence depends to a large degree on which wavefunction is used, whether it is enough or not to have necessary orbital interactions for certain electronic transitions to occur [7]. This may be explained by the coupling of the vibrational modes with the electronic transitions, which depend highly on the orbital interactions [8]. Thus, they need a model with an appropriate level of theory and large basis sets, where the polarization effect, representing the orbital interactions, is not negligible. Further discussion of this topic is presented in the next section. 


\subsection{Molecular Orbitals}

Figure 4 and 5 show the four main orbitals that contribute to the vertical electronic transitions in chlorophyll $a$, which were calculated at the basis set level of $6-31 \mathrm{G}(\mathrm{d}, \mathrm{p})$, using the RHF/CIS and TDDFT method respectively. These orbitals, HOMO-1, HOMO, LUMO and LUMO+1, represent the two highest occupied orbitals and the two lowest unoccupied orbitals in the chlorophyll $a$ molecule. Here, we only present the graphical representation of the calculated orbitals for the calculations using the highest-level basis set, 6$31 \mathrm{G}(\mathrm{d}, \mathrm{p})$, which gave the best fit between the UV-Vis spectra and the experimental spectra. This basis set includes the polarization effects of the unoccupied orbitals $d$ and $p$ on the occupied orbitals $p$ and $s$, respectively. Thus, the constructed molecular wavefunction has more sophisticated orbital interactions, which consequently provides more complete possible electronic transitions, as present in the calculated UV-Vis absorption spectra in the next section.

In general, if we compare the molecular orbitals presented in Figure 4 and 5, they provide almost the same spatial distribution of electrons in threedimensional space. Both calculations provide similar results, where the frontier orbitals of the molecules are centered on the porphyrin ring but not on the phytol chain. The consequence is that the structure of the porphyrin ring will be responsible for most of the chemical reactions and electronic transitions in the chlorophyll $a$ molecule, such as absorption, emission and charge transfer. The similar spatial distribution of orbital lobi between the pairs of HOMO/HOMO-1 and LUMO/LUMO+1, reveals the strong contribution of the structural symmetry of the porphyrin ring, which still exists even in the presence of functional groups such as a phytol chain, methine, methyl ester, methyl, ethyl and ethylene on the periphery of the ring. The similar spatial distribution between occupied and unoccupied frontier orbitals mostly contributes to the electronic transitions of the molecule involving photons that provide high oscillator strengths for both upward and downward transitions.

Figure 6 summarizes the energy levels of the main molecular orbitals that are involved in the electronic vertical transitions. Here, the TDDFT method provides more accurate results than the RHF/CIS method with respect to the experiment, where we have a strong absorption/emission in the red range $(\approx 2$ $\mathrm{eV})$. The RHF/CIS method tends to overestimate the energy gap between HOMO and LUMO. This can be improved slightly by increasing the polarization level of the basis set, which destabilizes HOMO faster than LUMO, and results in an increased energy gap. The same improvement can be used in the case of TDDFT calculations. Here, the effect is more pronounced because the LUMO energy is nearly independent of the polarization level, thus only 
HOMO is destabilized. Moreover, based on the MO analysis, we observed that at the basis set level of $6-31 \mathrm{G}(\mathrm{d}, \mathrm{p})$, the order of molecular orbitals around HOMO/LUMO will be independent of the level of calculation theory (Figure 4 and 5). This suggests that improvement by adjusting the polarization level of the basis set has reached saturation for this molecule. Thus, further improvement has to be achieved either by increasing the level of theory or the basis set level, e.g. by adding a localization or diffusion function.

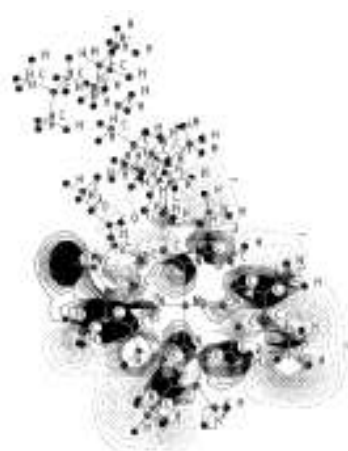

464 (LUMO)

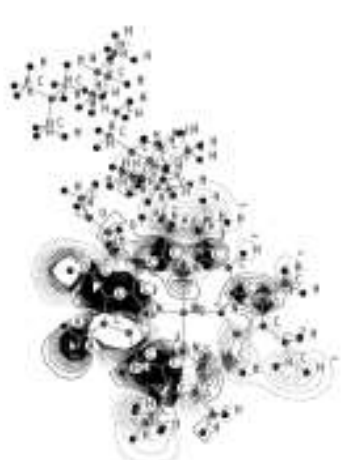

129 (HOMO)

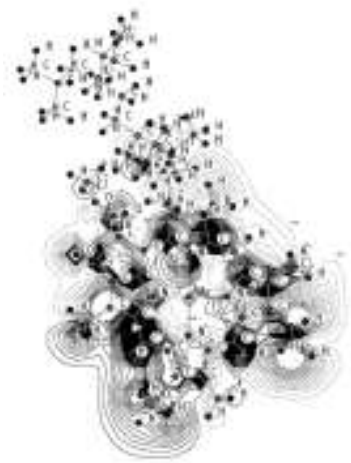

243 (LUMO+1)

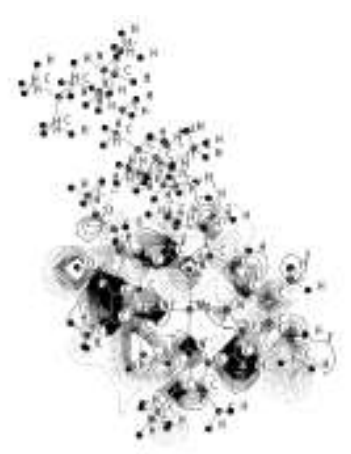

220 (HOMO-1)

Figure 4 The two highest occupied orbitals and the two lowest unoccupied orbitals in the chlorophyll $a$ molecule calculated with the RHF/CIS method using the $6-31 \mathrm{G}(\mathrm{d}, \mathrm{p})$ basis set.

Figure 6 also tells us that the RHF/CIS method tends to overestimate the interactions among AOs that form the frontier orbitals without involving long interactions among formed MOs. Thus, RHF/CIS gives better stabilization on HOMO and HOMO-1, but worse on LUMO and LUMO+1, resulting in an overestimated energy gap between HOMO and LUMO. TDDFT calculations in principle use the DFT method that has a delocalization feature of the molecular 
wavefunction due to the electron correlation effect of the exchange-correlation formulation [26-30]. Thus, DFT calculation naturally provides better long-range interactions among MOs, as presented by the less stabilized HOMO and HOMO-1, while LUMO and LUMO+1 are more stabilized, in comparison with RHF/CIS results. The delocalization feature of DFT is also obvious in the effect of polarization on the unoccupied orbitals, where the addition of polarization functions gives only a small contribution to the orbital stabilization. This may be explained by the nature of the unoccupied orbitals, which are more delocalized and spatially spread over a larger region, especially in the DFT formulation. This will reduce the effect of the polarization functions significantly. The polarization functions that act as a correction factor to add interaction between occupied and unoccupied orbitals in their AOs form, will lose their effect when the molecular wavefunctions get delocalized spatially. The delocalized wavefunctions may provide more interactions among MOs, which to some degree results in a better calculation of the electronic transitions compared to the experiment.

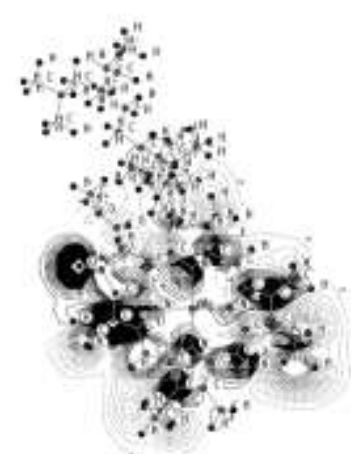

464 (LUMO)

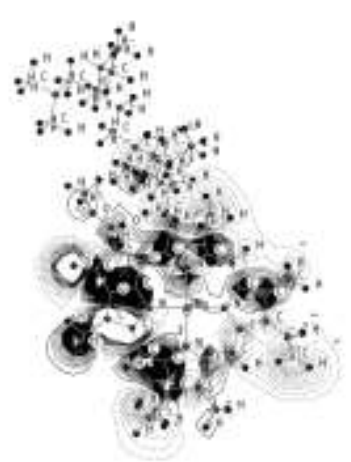

129 (НОМО)

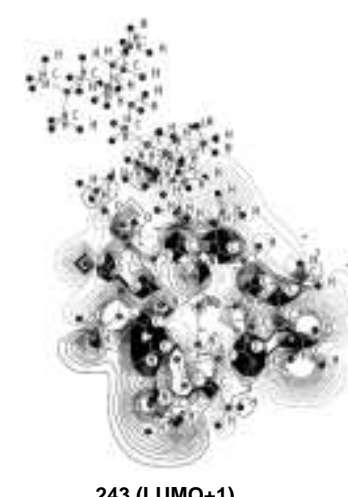

243 (LUMO+1)

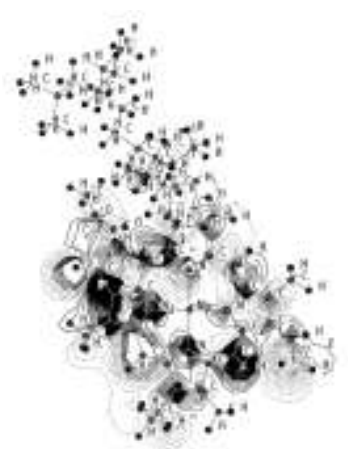

220 (HOMO-1)

Figure 5 The two highest occupied orbitals and the two lowest unoccupied orbitals in the chlorophyll $a$ molecule calculated with TDDFT using the 6$31 \mathrm{G}(\mathrm{d}, \mathrm{p})$ basis set. 


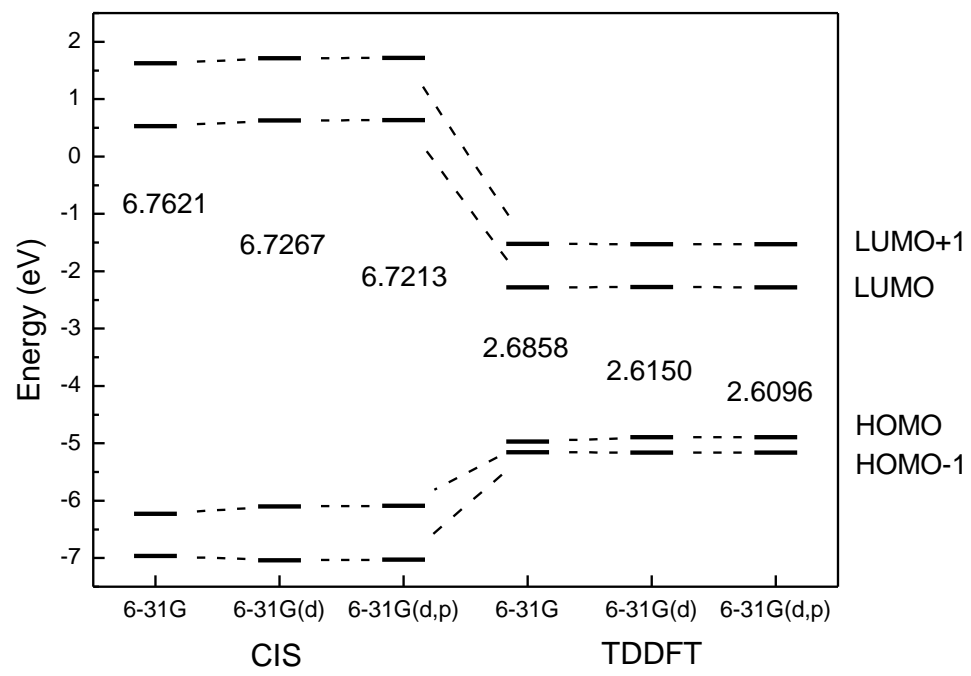

Figure 6 Energy levels of the two highest occupied orbitals and the two lowest unoccupied orbitals in the chlorophyll $a$ molecule calculated at different levels of theory and basis sets.

\subsection{Calculated UV-Vis Absorption Spectra}

Figure 7 shows the calculated UV-Vis absorption spectra of chlorophyll $a$ at different levels of theory and basis sets, which includes two semi-empirical methods, CNDO/S and ZINDO. All calculated spectra were corrected using their maximum transition with respect to the position of the $B$ transition of the Soret Band at $2.88 \mathrm{eV}$. The correction factor for each calculation is tabulated in Table 1. A correction factor closer to unity denotes a higher accuracy for the calculation method. All methods have a correction factor less than unity, which indicates the overestimation of the upward vertical electronic transition energies that are represented by the UV-Vis absorption spectra. In general, the semiempirical methods provide the best correction factors of all methods used in this study. This common feature is due to the nature of semi-empirical wavefunctions, which have been parameterized, especially in CNDO/S and ZINDO, for the study of electronic transitions involving photons [15-19]. 

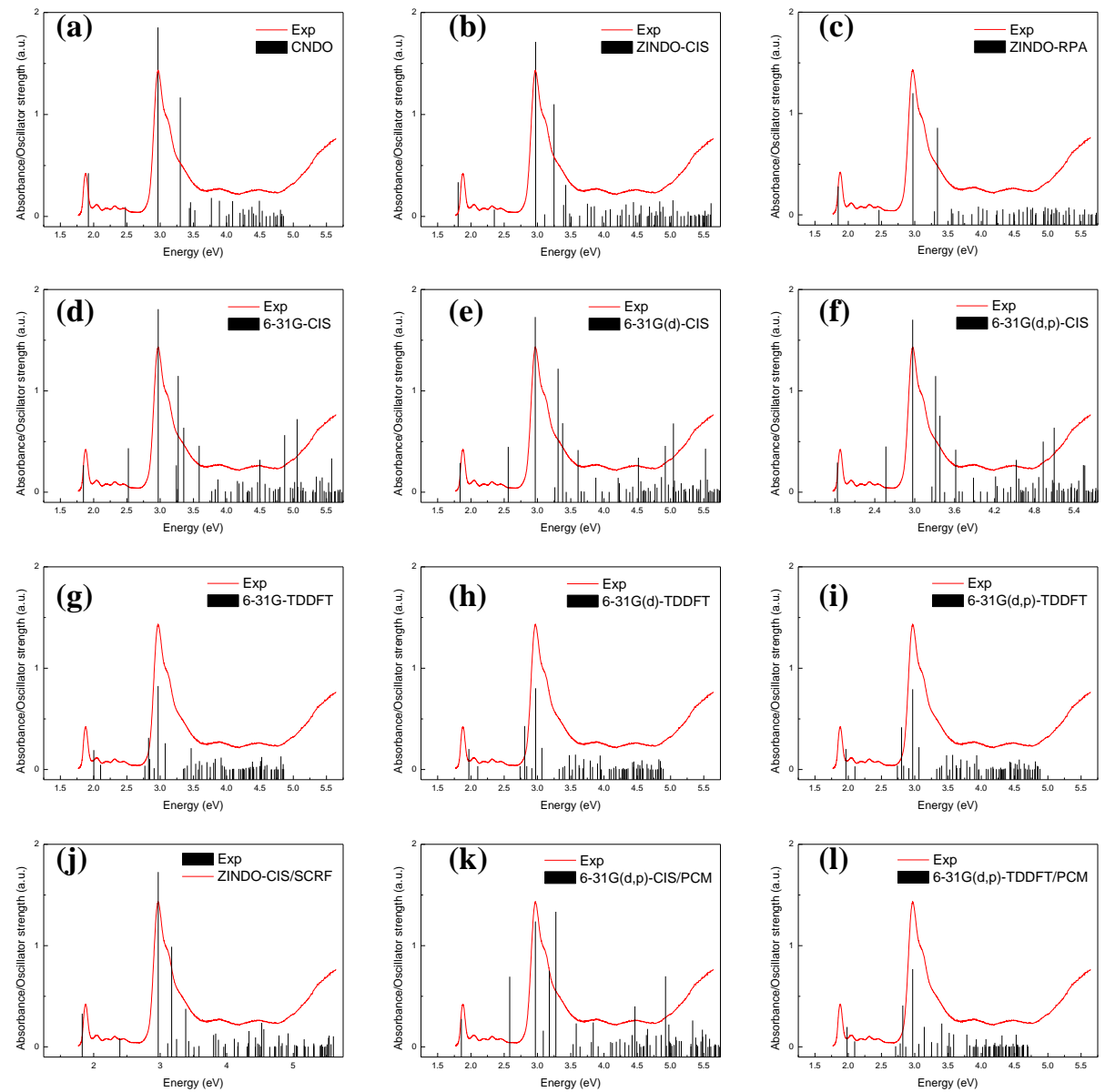

Figure 7 Calculated absorption spectra of the chlorophyll $a$ molecule at different levels of theory and basis sets: (a) CNDO/S, (b) ZINDO-CIS, (c) ZINDO-RPA, (d)RHF/CIS/6-31G, (e) RHF/CIS/6-31G(d), (f) RHF/CIS/631G(d,p), (g) TDDFT/ 6-31G, (h) TDDFT/6-31G(d), (i) TDDFT/6-31G(d,p), (j) ZINDO-CIS/SCRF(Methanol), (k) RHF/CIS/6-31G(d,p)/PCM(Methanol), and (l) TDDFT/6-31G(d,p)/ PCM(Methanol). All calculated spectra were corrected to the experimentally obtained spectrum of methanol, represented by the continuous curve [4].

In some cases, the use of semi-empirical methods suffices to calculate the UVVis absorption spectra, especially if they only need to provide the qualitative spectrum, consisting of the main electronic transitions. However, if we need to study the fine features of the electronic transitions, such as the transitions that appear between the $Q$ and the Soret bands in chlorophyll $a$, the ab initio methods are the better solution. Figure 7 compares the spectra calculated with all methods used in this study. It is obvious that ab intio methods, especially 
TDDFT and high-level polarization, provide more transition peaks in the region between the $Q$ and the Soret bands. As discussed in the previous section, the DFT treatment in principle also provides more orbital interactions than polarization functions, but in a much larger spatial region [26,27,30]. Based on their low occurrence, we predicted that the electronic transitions assigned to the fine features in the UV-Vis absorption spectra of chlorophyll $a$ have a low probability, due to the mismatch of the orbital symmetry. This effect can be reduced if the molecular wavefunctions become more loose and electrons are more delocalized- in other words, if the long interaction between MOs becomes more pronounced or stronger. This provides a condition that will not strictly preserve the symmetry during electronic transition due to the uncertainty of their distribution in space. Thus, both the DFT treatment and the addition of polarization functions in molecular wavefunctions may improve the calculation results. Moreover, this explanation is in a good agreement with the chemical nature of chlorophyll $a$, a molecule with many delocalized electrons, which provide more long-range interactions among MOs, which seems to be the origin of the fine features in the UV-Vis absorption spectra between the $Q$ and the Soret bands.

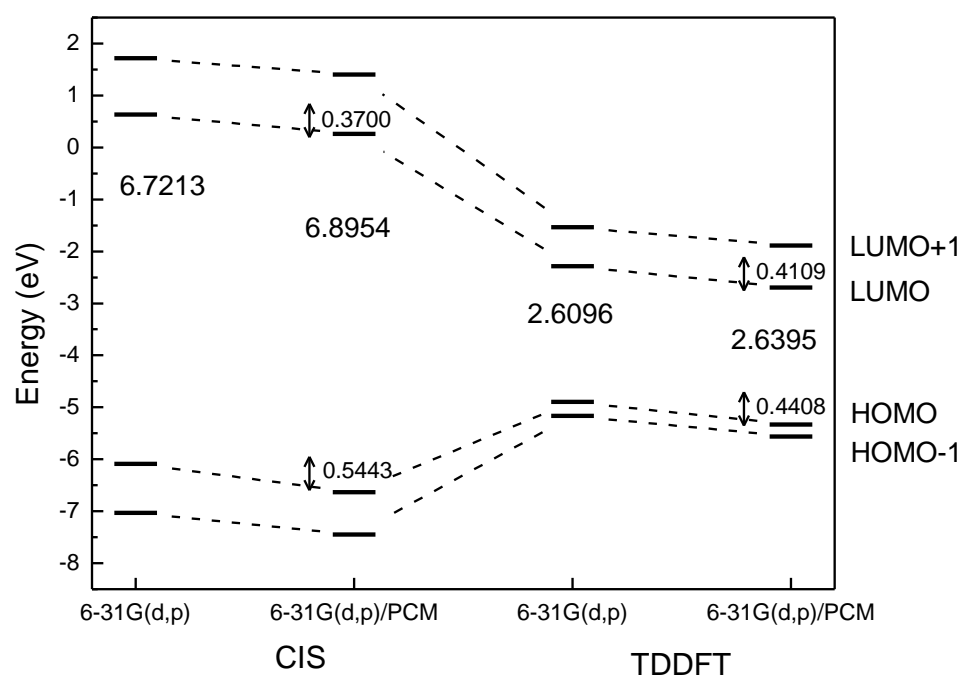

Figure 8 Effect of a solvent on the energy levels of the two highest occupied orbitals and the two lowest unoccupied orbitals in the chlorophyll $a$ molecule for TDDFT and RHF/CIS calculations in methanol based on the PCM model.

From the point of view of accuracy, the semi-empirical methods are still unmatched by the ab initio methods used in this study. However, as discussed 
above, the lack of fine features that may be important for certain purposes is their weak point. Here, the TDDFT methods provide the best results. As listed in Table 1 , the best TDDFT procedure used in this study, TDDFT/6-31G(d,p), has a correction factor very close to that of $\mathrm{CNDO} / \mathrm{S}$ but provides a more complete picture of the electronic transitions. This is reflected by the absorption fine features in Figure 7(i) in comparison with Figure 7(a). If we compare TDDFT/6-31G(d,p) with better semi-empirical methods, such as ZINDO-CIS or ZINDO-RPA, this method still provides more complete transitions, with a slightly lower correction factor (up to $9 \%$ ). Instead of its lower correction factor, it provides much more complete features, as presented in Figure 7(i) in comparison with Figure 7(b) and (c).

Tabel 1 Correction factor at different levels of theory and basis sets.

\begin{tabular}{lc}
\hline \multicolumn{1}{c}{ Method } & Correction factor \\
\hline CNDO/S & 0.846 \\
ZINDO-CIS & 0.867 \\
ZINDO-RPA & 0.922 \\
CIS/6-31G & 0.64 \\
TDDFT/6-31G & 0.836 \\
CIS/6-31G(d) & 0.645 \\
TDDFT/6-31G(d) & 0.8435 \\
CIS/6-31G(d,p) & 0.6475 \\
TDDFT/6-31G(d,p) & 0.844 \\
ZINDO-CIS/SCRF (Methanol) & 0.8598 \\
CIS/6-31G(d,p)/PCM (Methanol) & 0.6317 \\
TDDFT/6-31G(d,p)/PCM (Methanol) & 0.84 \\
\hline
\end{tabular}

Figure 7(j-1) shows the effect of methanol as a solvent on the calculated absorption spectra of chlorophyll $a$. Here, we compare three calculation methods: ZINDO-CIS, RHF/CIS and TDDFT, with SCRF and PCM solvation models, respectively. Table 1 shows that the presence of a solvation model in calculations of electronic transition spectra does not improve the correction factor. On the contrary, it tends to decrease the value of the correction factor, which means the solvation model overestimates the transition energies more. In this study, we will concentrate on the PCM model which is practically transferable and gives similar results compared to those of the SCRF based on the Onsager-Kirkwood formulation [31]. Figure 8 gives a clear illustration of the fact that the presence of a solvation model actually stabilizes the frontier orbitals. However, it stabilizes the occupied orbitals better than the virtual ones, 
i.e. in the case of TDDFT/6-31G(d,p) the PCM treatment stabilizes $0.4408 \mathrm{eV}$ for HOMO energy, but only $0.4109 \mathrm{eV}$ for LUMO. This leads to an increase in the electronic transition energies, which is reflected in a decrease of the correction factor value. This can be explained by the nature of the vertical electronic transition, which occurs very fast. According to the Franck-Condon principle, the vertical electronic transition occurs instantaneously from the occupied orbitals to the virtual orbitals, without any structural relaxation or interaction with its surroundings, i.e. structure relaxation, solvent polarization, etc., as a consequence of the Born-Oppenheimer approximation. As a simple illustration, the virtual orbitals can be constructed based on the occupied orbitals as in the CIS formulation (Eq. 3) or as unperturbed ground state density in the case of TDDFT (Eq. 10). Thus, the properties of the instantaneous virtual orbitals mostly depend on the features of the occupied or ground state orbitals. In a vertical transition, the solute electronic state changes via photon absorption or emission, which leads to a non-equilibrium response of the solvent that only involves solvent electrons [22,32]. In other words, the solvent electronic polarization is in equilibrium with the excited-state electron density of the solute, while its structural properties remain equilibrated with the ground state electron density. In this study, the starting structure for the PCM treatment was optimized in vacuum, therefore this will lead to an error with respect to the occupied orbitals, which propagates to a larger error with respect to the virtual orbitals.

In the PCM treatment, the solute-solvent interactions are described in terms of a solvent reaction potential, $\hat{V}_{\text {int }}$, which is introduced as a perturbation of the Hamiltonian of the isolated molecule, $\hat{H}^{0}[33]$ :

$$
\hat{H}_{\text {eff }}=\hat{H}^{0}+\hat{V}_{\text {int }}
$$

Thus, in this formulation, the properties of the solute-solvent interactions of the ground state molecule are still valid to be calculated using the ground state wavefunction optimized in vacuum, but not in the case of excited molecules. In an excited molecule, a correct ground state wavefunction optimized for an appropriate solvent is required, which leads to a correct projection of the virtual orbitals that will be well-stabilized, just like the occupied orbitals in the PCM treatment.

\section{Conclusions}

In this work, the vertical electronic transitions of chlorophyll $a$ were calculated using semi-empirical and ab initio methods to provide both contributed molecular orbitals and UV-Vis absorption spectra. The SAAP (Spin-Adapted 
Antisymmetrized Product) treatment of both RHF/CIS and TDDFT calculations reveals that the main molecular orbitals contributing to the electronic transitions are HOMO-1, HOMO, LUMO and LUMO+1, independent of the level of theory and basis sets. In this study, both the addition of polarization functions and DFT treatment in molecular wavefunctions increase the accuracy of the calculated UV-Vis absorption spectra. This can be concluded due to the increase of spatially long-range MO interactions. Here, the effect of the DFT treatment is more pronounced due to the delocalized nature of their wavefunctions. The effect of the addition of polarization functions is more obvious in the RHF/CIS treatment due to their localized wavefunctions. TDDFT/6-31G(d,p), the procedure with the highest level of theory in this study, provides more complete transitions with a slightly lower correction factor (up to $9 \%$ ) compared to ZINDO-RPA. The present solvation model, i.e. PCM, stabilizes the occupied orbitals better than the virtual ones for the starting structure optimized in vacuum, due to the nature of its mathematical formulation. This suggests structure optimization in an appropriate solvent prior to any PCM calculation for excited-state properties.

\section{Acknowledgements}

This work was supported by Riset KK-ITB Grant Project 2009 with contract number 270/K01.7/PL/2009. The authors would like to thank to Alex A. Granovsky, Department of Chemistry, Moscow State University, B. Prijamboedi, and M.A. Martoprawiro, Department of Chemistry, Institut Teknologi Bandung, for various discussions and helpful suggestions about the ab initio calculation of electronic transition spectra. V. Suendo acknowledges partial funding from Riset KK-ITB Grant Project 2011 with contract number 225/I.1.C01/PL/2011.

\section{References}

[1] Barazzouk, S. \& Hotchandani, S., Enhanced Charge Separation in Chlorophyll A Solar Cell by Gold Nanoparticle, J. Appl. Phys., 96, pp. 7744-7746, 2004.

[2] Shimatani, K., Tajima, H., Komino, T., Ikeda, S., Matsuda, M., Ando, Y. \& Akiyama, H., The Electroluminescence Spectrum of Chlorophyll a, Chem. Lett., 34, pp. 948-949, 2005.

[3] Linnanto, J. \& Korppi-Tommola, J., Spectroscopic Properties of MgChlorin, Mg-Porphin and Chlorophylls $a, b, c 1, c 2, c 3$ and $d$ Studied by Semi-Empirical And Ab Initio MO/CI Methods, Phys. Chem. Chem. Phys., 2, pp. 4962-4970, 2000. 
[4] Du, H., Fuh, R.-C., Li, J.Z., Corkan, L.A. \& Lindsey, J.S., PhotochemCAD HD 1.1, Carnegie Mellon University and North Carolina State University, 1998.

[5] Vokacova, Z. \& Burda, J.V., Computational Study on The Spectral Properties of The Selected Pigments From Various Photosystems: Structure-Transition Energy Relationship, J. Phys. Chem. A, 111, pp. 5864-5878, 2007.

[6] Hasegawa, J., Ozeki, Y., Ohkawa, K. \& Nakatsuji, H., Theoretical Study of the Excited States of Chlorin, Bacteriochlorin, Pheophytin a, and Chlorophyll a by the SAC/SAC-CI Method, J. Phys. Chem. B, 102, pp. 1320-1326, 1998.

[7] Sundholm, D., Density Functional Theory Calculations of The Visible Spectrum of Chlorophyll a, Chem. Phys. Lett., 302, pp. 480-484, 1999.

[8] Sundholm, D., Comparison of the Electronic Excitation Spectra of Chlorophyll $a$ and Pheophytin a Calculated at Density Functional Theory Level, Chem. Phys. Lett., 317, pp. 545-552, 2000.

[9] Parusel, A. \& Grime, S., DFT/MRCI Calculations on The Excited States of Porphyrin, Hydroporphyrins, Tetrazaporphyrins and Metalloporphyrins, J. Porphyrins Phthalocyanines, 5, pp. 225-232, 2001.

[10] Dreuw, A. \& Head-Gordon, M., Single-Reference ab Initio Methods for the Calculation of Excited States of Large Molecules, Chem. Rev., 105, pp. 4009-4037, 2005.

[11] Staemmler, V., Introduction to Hartree-Fock and CI Methods, In J. Grotendorst, S. Blügel, D. Marx (Eds.), Computational Nanoscience: Do It Yourself!, John von Neumann Institute for Computing, Jülich, NIC Series, 31, pp. 1-18, 2006.

[12] Rohringer, N., Peter, S. \& Burgdörfer, J., Calculating State-To-State Transition Probabilities within Time-Dependent Density-Functional Theory, Phys. Rev. A, 74, 042512-1-7, 2006.

[13] Doltsinis, N.L., Time-Dependent Density Functional Theory, In J. Grotendorst, S. Blügel, D. Marx (Eds.), Computational Nanoscience: Do It Yourself!, John von Neumann Institute for Computing, Jülich, NIC Series, 31, pp. 357-373, 2006.

[14] Granovsky, A.A., PC GAMESS/Firefly version 7.1.G, www http://classic.chem.msu.su/gran/gamess/index.html (December $4^{\text {th }}, 2009$ ).

[15] DelBene, J. \& Jaffe, H.H., Use of the CNDO Method in Spectroscopy. I. Benzene, Pyridine, and the Diazines, J. Chem. Phys., 48, pp. 1807-1813, 1968.

[16] DelBene, J. \& Jaffe, H.H., Use of the CNDO Method in Spectroscopy. II. Five - Membered Rings, J. Chem. Phys., 48, pp. 4050-4055, 1968. 
[17] Ridley, J. \& Zerner, M.C., An Intermediate Neglect of Differential Overlap Technique for Spectroscopy: Pyrrole and the Azines, Theor. Chim. Acta, 32, pp. 111-134, 1973.

[18] Thompson, M. \& Zerner, M.C., A Theoretical Examination of The Electronic Structure and Spectroscopy of The Photosynthetic Reaction Center From Rhodopseudomonas Viridis, J. Am. Chem. Soc., 113, pp. 8210-8215, 1991.

[19] Bacon, A.D. \& Zerner, M.C., An Intermediate Neglect of Differential Overlap Theory for Transition Metal Complexes: $\mathrm{Fe}$, Co and $\mathrm{Cu}$ Chlorides, Theor. Chim. Acta, 53, pp. 21-54, 1979.

[20] Karelson, M.M. \& Zerner, M.C., Theoretical Treatment of Solvent Effects on Electronic Spectroscopy, J. Phys. Chem., 96, pp. 6949-6957, 1992.

[21] Mennucci, B. \& Tomasi, J., Continuum Solvation Models: a New Approach to the Problem of Solute's Charge Distribution and Cavity Boundaries, J. Chem. Phys., 106, pp. 5151-5158, 1997.

[22] Tomasi, J., Mennucci, B. \& Cammi, R., Quantum Mechanical Continuum Solvation Models, Chem. Rev., 105, pp. 2999-3093, 2005.

[23] Klamt, A., Mennucci, B., Tomasi, J., Barone, V., Curutchet, C., Orozco, M. \& Javier Luque, F., On the Performance of Continuum Solvation Methods. A Comment on "Universal Approaches to Solvation Modelling”, Acc. Chem. Res., 42, pp. 489-492, 2009.

[24] Gouterman, M., Spectra of Porphyrins, J. Mol. Spectrosc., 6, pp. 138163, 1961.

[25] Fajer, J., Chlorophyll Chemistry Before and After Crystals of Photosynthetic Reaction Centers, Photosynthesis Research, 80, pp. 165172, 2004.

[26] Gritsenko, O.V. \& Baerends, E.J., Electron Correlation Effects on the Shape of Kohn-Sham Molecular Orbital, Theor. Chem. Acc., 96, pp. 4450, 1997.

[27] Poater, J., Sola, M., Duran, M. \& Fradera, X., The Calculation of Electron Localization and Delocalization Indices at The Hartree-Fock, Density Functional and Post-Hartree-Fock Levels of Theory, Theor. Chem. Acc., 107, pp. 362-371, 2002.

[28] Mori-Sanchez, P., Cohen, A.J. \& Yang, W., Many-Electron SelfInteraction Error in Approximate Density Functionals, J. Chem. Phys., 125, p. 201102, 2006.

[29] Cohen, A.J., Mori-Sanchez, P. \& Yang, W., Development of ExchangeCorrelation Functionals with Minimal Many-Electron Self-Interaction Error, J. Chem. Phys., 126, p. 191109, 2007.

[30] Cohen, A.J., Mori-Sanchez, P. \& Yang, W., Insights into Current Limitations of Density Functional Theory, Science, 321, pp. 792-794, 2008. 
[31] Curutchet, C., Cramer, C.J., Truhlar, D.G., Ruiz-Lopez, M.F., Rinaldi, D., Orozco, M. \& Luque, F.J., Electrostatic Component of Solvation: Comparison of SCRF Continuum Models, J. Comp. Chem., 24, pp. 284297, 2003.

[32] Improta, R., Barone, V., Scalmani, G. \& Frisch, M.J., A State-Specific Polarizable Continuum Model Time Dependent Density Functional Theory Method for Excited State Calculations in Solution, J. Chem. Phys., 125, p. 054103, 2006.

[33] Caricato, M., Mennucci, B. \& Tomasi, J., Solvent Effects on The Electronic Spectra: An Extension of The Polarizable Continuum Model to The ZINDO Method, J. Phys. Chem. A, 108, pp. 6248-6256, 2004. 